\title{
Mode of delivery and breastfeeding practices
}

\begin{abstract}
Background: We compared early breastfeeding practices, including initiation, exclusivity, and intensity among women who had an unplanned caesarean section (c-section), a planned c-section, vaginal induced delivery, and a vaginally but not induced delivery.
\end{abstract}

Methods: This was a retrospective study involving secondary data analysis using the Infant Feeding Practices Study II, a longitudinal national survey, which followed women from pregnancy to 1 year postpartum. Data were obtained from the prenatal, neonatal and 2-month surveys for 2541 mothers and their infants. Descriptive statistics and chisquare analysis were conducted to identify any associations between mode of delivery and maternal characteristics, breastfeeding patterns in the first two months of infancy, prenatal breastfeeding attitudes, sources of breastfeeding information and reasons for not initiating breastfeeding.

Results: Women with a planned c-section had a significantly lower breastfeeding initiation rate $(80 \%)$. Further, they were least likely to report a clinician's support for breastfeeding $(40.2 \%)$ and more likely to disagree with benefits of breastfeeding $(26.3 \%)$. Those with vaginally non-induced deliveries were least likely to receive breastfeeding information during the postpartum period from lactation consultants $(56.5 \%)$, while women who had a planned c-section were less likely to receive breastfeeding information from relatives/ friends $(48.9 \%)$.

Conclusion: Findings suggest that breastfeeding practices vary considerably by mode of delivery and this should be taken into consideration when offering lactation services and information to women. Women may need encouragement from family members and clinicians in initiating breastfeeding as well as adequate breastfeeding support through month 2 postpartum.

Keywords: breastfeeding, breastfeeding initiation, cesarean section, pregnancy, prenatal, vaginal birth, planned and unplanned cesarean section
Volume 2 Issue $6-2017$

\author{
Hira Palla and Panagiota Kitsantas \\ Department of Health Administration and Policy, George Mason \\ University, USA \\ Correspondence: Panagiota Kitsantas, Professor of \\ Biostatistics and Epidemiology, College of Health and Human \\ Services, George Mason University, 4400 University Drive, MS \\ IJ3 Fairfax,VA 22030, USA, Tel +703 993-9164, \\ Email pkitsant@gmu.edu
}

Received: July 10, 2017 | Published: October 06, 2017

\section{Introduction}

Despite the well-known benefits of nourishing infants exclusively with mother's milk, the rates of breastfeeding initiation, continuation, and exclusive breastfeeding during the first few months of infancy are less than ideal among US mothers. ${ }^{1,2}$ Mothers' and infants' experiences during labor and delivery may affect lactation and breastfeeding outcomes. Vaginally induced birth has been associated with not initiating breastfeeding. ${ }^{1}$ Furthermore, cesarean deliveries (c-sections) have been associated with lower initiation rates and shorter breastfeeding duration compared to undergoing a vaginal delivery. ${ }^{3,4}$ This may be due to delays in mother/infant skin-to-skin contact, mother's post-surgery physical complications, and effects of anesthetics. ${ }^{5-7}$ In addition to mode of delivery, other factors that may influence breastfeeding initiation and duration include challenges encountered during nursing, availability of support systems, mother's socio-cultural and economic situation, and the recommendations and attitudes of family, peers, and healthcare professionals. ${ }^{8-11}$

Previous studies that have examined the relationship between mode of delivery and breastfeeding outcomes had either small sample sizes or were confined to a single geographic location. ${ }^{12}$ Further, findings from studies examining mode of delivery and breastfeeding are inconsistent. ${ }^{12}$ The purpose of the present study was to evaluate early breastfeeding practices, including initiation, exclusivity, and intensity of breastfeeding in the first 2 months of infant age among women who had an unplanned c-section, a planned c-section, vaginal induced delivery, and a vaginally but not induced delivery using a large, nationally distributed US sample. Further, we assessed relationships between breastfeeding support by family, peers, clinicians, resources of breastfeeding information and reasons for not initiating breastfeeding by mode of delivery.

\section{Methods}

Data were obtained from the Infant Feeding Practices Study (IFPS II), a longitudinal national survey administered by the U.S. Food and Drug Administration and Centers for Disease Control and Prevention that followed women from pregnancy to 1 year postpartum. The IFPS II data were collected between 2005 and 2007 using one prenatal and 10 postnatal questionnaires that primarily assessed infant feeding practices and infant anthropometric characteristics. Approximately 4900 women participated at the early phases of the IFPS II study. Although this is a national sample of women and their infants, it is not representative of the U.S. population of mothers/infants. In IFPS II, inclusion criteria required that the infant was born after 35 weeks gestation, weighed at least $5 \mathrm{lbs}$., was a singleton, and infant did not require hospitalization longer than 3 days following birth. ${ }^{13}$ In the present study, we used information from the prenatal, neonatal and 2-month infant age surveys. The sample size for the current study included 2541 mothers and their infants who had complete data on the outcome variables considered in this study. Of the 2541 women, 418 never initiated breastfeeding. This group of 418 women was further analyzed to assess reasons for not initiating breastfeeding by mode of delivery. 
Mode of delivery, which included the categories of vaginally not induced labor, vaginally induced labor, planned c-section, and unplanned c-section, was the stratification variable. The outcome variable included breastfeeding patterns during the first two months (baby was ever breastfed, exclusive breastfeeding at infant age two months, and breastfeeding intensity). Mothers were asked during the neonatal survey if they initiated breastfeeding. This question led to the creation of the breastfeeding initiation variable (yes/no). Exclusive breastfeeding (yes/no) at infant age two months was defined as complete infant nutrition through breast milk without any other nutritional supplementation for the infant with the exception of liquid vitamin supplements and up to four ounces of water per day. Breastfeeding intensity was calculated by averaging the proportion of breast milk to the total milk diet (including breast milk, formula, cow's milk, other milk) that the baby received daily; a categorical variable was created with two levels: low defined as $<20 \%$ of milk feedings being breast milk, and medium/high defined as $\geq 20 \%$ of milk feedings being breast milk. The construction of breastfeeding intensity was based on previous research that used the same IFPS II data to assess breastfeeding practices..$^{14,15}$

Family member and clinician opinions pertaining to infant feeding were assessed during the prenatal period by inquiring about the mother's, her partner's, and her clinician's beliefs regarding the best method to feed the baby. Family and clinician opinion included three categories according to the preferred infant feeding method: breastfeed only, formula only or both formula and breastfeed, and no opinion. Due to low reported rates on the clinician and family opinion, the category of both formula and breastfeed was combined with formula only to create the category formula only or both (meaning breastfeeding and formula). Further, mother's opinion regarding breastfeeding benefits was assessed via a number of questions about various benefits observed in breastfed babies. These included decreased incidence of diarrhea, decreased ear infections and upper respiratory infections, and lower obesity rates in breastfed babies. Information was obtained using a Likert scale (disagree, no opinion and agree). Mother's plan regarding feeding practices was assessed during the prenatal period. Responses were grouped into breastfeeding only, formula only, formula and breast milk combined, and don't know.

Mothers were asked during the postpartum period at 2 monthsinfant age if they received breastfeeding information from sources which were presented to the participants as follows: lactation consultant, relatives/friends, birthing or baby care class, and nurse/ nurse midwife/nurse practitioner. This question led to the creation of the four sources of breastfeeding information variables (yes/no).

We assessed several reasons for not initiating breastfeeding among those mothers who responded that they never breastfed their infants during the neonatal period. Reasons for not initiating breastfeeding included medical reasons, mother finding formula to be the same or better than breast milk, inconvenience, wanting to leave baby hours at a time, lifestyle changes, having work or school, someone else fed the baby, and family reasons. Medical reasons included mother being sick or on medication. Questions on whether the mother wanted to go on a weight loss diet, go back to usual diet, smoke, wanted body back to herself, and wanted to use incompatible contraception were all classified as lifestyle changes and combined to create the variable, lifestyle changes with two categories (one to two and three or more lifestyle changes). Questions about whether the father or grandmother of the infant did not want the mother to breastfeed were combined to create the variable, family reasons.

Several sociodemographic characteristics were used to describe the sample, including mother's age $(18-24,25-34$, and $>34$ years old), mother's education (high school or less, some college, 4-year college or more) and marital status (married, widowed/divorced/ separated, never married). Prenatal smoking (non-smoker, smoker) and prepregnancy BMI ( $<19.8$ underweight, 19.8 to $\leq 26$ normal, $>26$ to $\leq 29$ overweight, and $>29$ obese).

\section{Data analysis}

Descriptive statistics and chi-square analysis were conducted to identify any associations between mode of delivery and maternal characteristics, breastfeeding patterns in the first two months of infancy, prenatal breastfeeding attitudes, sources of breastfeeding information and reasons for not initiating breastfeeding. A p-value $<0.05$ was considered statistically significant.

\section{Results}

The sample included 2541 women. Approximately 16.5\% (418 women) of this sample reported that they never breastfed. Table 1 presents the distribution of prenatal maternal characteristics by mode of delivery. We observed that women who were older than 34 years were more likely to have a planned c-section $(23.7 \%)$ and less likely to have a vaginally induced delivery $(11.5 \%)$. Women with a planned c-section were significantly more likely to be obese $(41.4 \%)$ and married $(85.6 \%)$. On the other hand, women who had an unplanned c-section were more likely to have never been married (23.8\%). Prenatal smoking was associated with a higher likelihood of having an unplanned c-section (11\%). A significantly larger percent of women who underwent a vaginally not induced labor had a normal BMI (51.7\%).

Table 2 describes associations between breastfeeding opinions and mode of delivery. A significantly higher proportion of women with a non-induced vaginal delivery had families who supported breastfeeding only $(52.4 \%)$. Women who had a planned c-section had a higher likelihood of having family members $(40.8 \%)$ and clinicians $(20.9 \%)$ supporting formula only or the use of a combination of formula and breast milk. Women with a vaginally not induced delivery were significantly more likely to agree with breastfeeding benefits $(41.6 \%)$ compared to the other groups. Women who underwent a planned c-section were less likely to report agreement with breastfeeding benefits; around $26 \%$ reported disagreement with benefits of breastfeeding. Further, this group was also significantly less likely to report prenatally a plan to exclusively breastfeed their child $(52.1 \%)$ and more likely to indicate their plan to feed their infant formula only (18.8\%) in comparison to the other modes of delivery groups.

Table 3 presents breastfeeding patterns at 2-months infant age stratified by mode of delivery. Twenty percent of women who had a planned c- section did not initiate breastfeeding. Furthermore, women who had an unplanned c-section were significantly less likely to exclusively breastfeed their baby at two months (30.5\%). Among women who breastfed their baby at two months, a higher proportion of those who underwent vaginally induced labor had the lowest breastfeeding intensity $(35.6 \%)$, followed closely by women who had an unplanned c-section (35.1\%).

A significantly lower proportion (48.9\%) of women with a planned c-section received breastfeeding information from relatives/friends (table 4). Women who underwent vaginally induced labor were significantly less likely to be provided with breastfeeding information from both a nurse/midwife/nurse practitioner (59.3\%) and a birthing 
or baby care class $(29.4 \%)$.

Table I Distribution of prenatal sample characteristics by mode of delivery

\begin{tabular}{|c|c|c|c|c|c|}
\hline Characteristics & $\begin{array}{l}\text { Vaginally not } \\
\text { induced \% }\end{array}$ & $\begin{array}{l}\text { Vaginally \& } \\
\text { induced \% }\end{array}$ & $\begin{array}{l}\text { Planned } \\
\text { C-section \% }\end{array}$ & $\begin{array}{l}\text { Unplanned } \\
\text { C-section \% }\end{array}$ & P-value \\
\hline \multicolumn{6}{|l|}{ Mother's age } \\
\hline 18-24 years & 60.4 & 64.8 & 64 & 51 & \\
\hline $25-34$ years & 25.5 & 23.7 & 12.3 & 28.4 & 0.00 \\
\hline$>34$ years & 14 & 11.5 & 23.7 & 20.7 & \\
\hline \multicolumn{6}{|l|}{ Mother's education } \\
\hline High school or less & 20.5 & 23.4 & 18.8 & 18.7 & \\
\hline Some college & 39.3 & 40.8 & 41.7 & 39.9 & 0.20 \\
\hline $\begin{array}{l}\text { 4-year college or } \\
\text { More }\end{array}$ & 40.2 & 38.5 & 39.5 & $4 \mathrm{I} .4$ & \\
\hline \multicolumn{6}{|l|}{ Marital status } \\
\hline Married & 77.6 & 79.8 & 85.6 & 72.4 & \\
\hline Widowed/Divorced/ & 4.5 & 4.7 & 4.1 & 3.7 & 0.00 \\
\hline \multicolumn{6}{|l|}{ Separated } \\
\hline Never Married & 17.9 & 15.5 & 10.3 & 23.8 & \\
\hline \multicolumn{6}{|l|}{ Prenatal smoking } \\
\hline Non-smoker & 90.2 & 90.5 & 90.1 & 89 & \\
\hline Smoker & 9.8 & 9.5 & 9.9 & 11 & 0.85 \\
\hline \multicolumn{6}{|l|}{ Prepregnancy BMI } \\
\hline Normal & 51.7 & 47.3 & 36.2 & 38.4 & \\
\hline Underweight & 13.9 & 10.8 & 7.5 & 5.3 & \\
\hline Overweight & 14.4 & 14.8 & 15 & 17.9 & 0.00 \\
\hline Obese & 20.1 & 27.2 & 41.4 & 38.4 & \\
\hline
\end{tabular}

Table 2 Breast feeding opinions during the prenatal period by mode of delivery

\begin{tabular}{|c|c|c|c|c|c|}
\hline $\begin{array}{l}\text { Breastfeeding opinions in } \\
\text { prenatal period }\end{array}$ & $\begin{array}{l}\text { Vaginally not } \\
\text { induced \% }\end{array}$ & $\begin{array}{l}\text { Vaginally \& } \\
\text { induced \% }\end{array}$ & $\begin{array}{l}\text { Planned } \\
\text { C-section \% }\end{array}$ & $\begin{array}{l}\text { Unplanned } \\
\text { C-section \% }\end{array}$ & P-value \\
\hline \multicolumn{6}{|c|}{ Clinician's opinion about infant feeding } \\
\hline Breastfeeding only & 48.1 & 45.5 & 40.2 & 46.4 & \\
\hline Formula only or both & 14.6 & 15.8 & 20.9 & 15 & 0.02 \\
\hline No opinion & 37.3 & 38.7 & 38.8 & 38.6 & \\
\hline \multicolumn{6}{|c|}{ Family's opinion about infant feeding } \\
\hline Breastfeeding only & 52.4 & 51.2 & 42.5 & 49.3 & \\
\hline Formula only or both & 32.4 & 33.6 & 40.8 & 34.3 & 0.01 \\
\hline No opinion & 15.1 & 15.2 & 16.7 & 16.4 & \\
\hline \multicolumn{6}{|c|}{ Mother's opinion about breastfeeding benefits } \\
\hline Agree & 41.6 & 36.1 & 36 & 39.8 & \\
\hline Disagree & 19.8 & 19.8 & 26.3 & 19.6 & 0.02 \\
\hline No opinion & 38.5 & 41.1 & 37.7 & 40.6 & \\
\hline \multicolumn{6}{|l|}{ Plan to feed the baby } \\
\hline Breastfeeding only & 62.7 & 60.1 & 52.1 & 60.5 & \\
\hline Formula only & 12.2 & 12.2 & 18.8 & 12.7 & 0.00 \\
\hline Breastfeeding \& Formula & 21.8 & 24.9 & 24.5 & 22.7 & \\
\hline Don't know & 3.3 & 2.9 & 4.5 & 4.1 & \\
\hline
\end{tabular}

aPlans for feeding the infant based on maternal responses during the prenatal period 
Table 3 Breastfeeding patterns at 2 months postpartum by mode of delivery

\begin{tabular}{lcllll}
\hline $\begin{array}{l}\text { Breastfeeding } \\
\text { patterns }\end{array}$ & $\begin{array}{c}\text { Vaginally not } \\
\text { induced \% }\end{array}$ & $\begin{array}{l}\text { Vaginally \& } \\
\text { induced \% }\end{array}$ & $\begin{array}{l}\text { Planned } \\
\text { C-section \% }\end{array}$ & $\begin{array}{l}\text { Unplanned } \\
\text { C-section \% }\end{array}$ & P-value \\
\hline $\begin{array}{l}\text { Baby was ever breastfeed } \\
\text { Breastfed }\end{array}$ & 87 & 85.4 & 80 & 86 & 0.00 \\
Not Breastfed & 13 & 14.6 & 20 & 14 & \\
Exclusive breastfeeding & 44.7 & 34.3 & 36.3 & 30.5 & 0.00 \\
Yes & 55.3 & 65.7 & 63.7 & 69.5 & \\
No & & & & \\
Breastfeeding intensity & 24.7 & 35.6 & 34.3 & 35.1 & 0.00 \\
Low & 75.3 & 64.4 & 65.7 & 64.9 & \\
Medium/High & & & & \\
\hline
\end{tabular}

Table 5 presents information about the reasons for not initiating breastfeeding in the neonatal period by mode of delivery. These findings pertain only to the 418 mothers who did not initiate breastfeeding. Women who had an unplanned c-section were more likely to cite medical issues $(45.8 \%)$ and having work or school (59.6\%). While, women who underwent a vaginally not induced delivery cited inconvenience $(58.6 \%)$, wanting to leave baby hours at a time (45.7\%), finding formula to be the same or better compared to breast milk (71\%), wanting someone else to feed the baby (55\%), and discouragement from family members $(16.9 \%)$ as important reasons. A higher proportion $(16 \%)$ of women who underwent vaginally induced labor reported at least three lifestyle changes as important reasons for not initiating breastfeeding.

Table 4 Sources of breastfeeding information assessed at 2 months postpartum by mode of delivery

\begin{tabular}{llllll}
\hline $\begin{array}{l}\text { Sources of breastfeeding } \\
\text { information }\end{array}$ & $\begin{array}{l}\text { Vaginally not } \\
\text { induced \% }\end{array}$ & $\begin{array}{l}\text { Vaginally \& } \\
\text { induced \% }\end{array}$ & Planned C-section \% & $\begin{array}{l}\text { Unplanned } \\
\text { C-section \% }\end{array}$ & P-value \\
\hline $\begin{array}{l}\text { Nurse/nurse midwife/Nurse } \\
\text { practitioner }\end{array}$ & 64.4 & 59.3 & 61.3 & 69.3 & 0.02 \\
Lactation consultant & 56.5 & 61.4 & 63 & 70.3 & 0.00 \\
Relatives/friends & 56 & 51 & 48.9 & 62.5 & 0.00 \\
Birthing or baby care class & 35 & 29.4 & 32 & 40.3 & 0.01 \\
\hline
\end{tabular}

Table 5 Reasons for not initiating breastfeeding in the neonatal period by mode of delivery

\begin{tabular}{|c|c|c|c|c|c|}
\hline Reasons & $\begin{array}{l}\text { Vaginally not } \\
\text { induced \% }\end{array}$ & $\begin{array}{l}\text { Vaginally \& } \\
\text { induced \% }\end{array}$ & $\begin{array}{l}\text { Planned c-section } \\
\%\end{array}$ & $\begin{array}{l}\text { Unplanned } \\
\text { c-section \% }\end{array}$ & P-value \\
\hline Medical Reasons ${ }^{a}$ & 26.4 & 32.6 & 29.9 & 45.8 & 0.09 \\
\hline Formula is same or better & 71 & 57.2 & 69.3 & 61.2 & 0.07 \\
\hline Inconvenience & 58.6 & 51.8 & 48.2 & 49 & 0.40 \\
\hline $\begin{array}{l}\text { Wanted to leave baby hours } \\
\text { at a time }\end{array}$ & 45.7 & 41.9 & 31.4 & 29.4 & 0.06 \\
\hline Have work or school & 46.5 & 40.3 & 34.1 & 59.6 & 0.02 \\
\hline Someone else feed the baby ${ }^{b}$ & 55 & 51.1 & 40.7 & 54.2 & 0.19 \\
\hline Family reasons ${ }^{c}$ & 16.9 & 11.9 & 10.3 & 4 & 0.09 \\
\hline \multicolumn{6}{|l|}{ Lifestyle changes $^{d}$} \\
\hline I-2 lifestyle changes & 37 & 32.1 & 25.9 & 20.4 & 0.15 \\
\hline 3 or more lifestyle changes & 15.2 & 16 & 10.6 & 12.2 & \\
\hline
\end{tabular}

aMedical Reasons includes medical reasons and mom was sick or on medication.

bSomeone else feed the baby includes wanted someone else to feed baby and someone else wanted to feed baby. 'Family reasons include baby's grandmother didn't want her to breastfeed and baby's father didn't want her to breastfeed.

¿Lifestyle changes include wanted to go on a weight loss diet, go back to original diet, wanted to smoke, mom wanted body back to herself, wanted to use incompatible contraception. 


\section{Discussion}

In the present study, although the majority of the women indicated prenatally their plan to only breastfeed their infant, these rates diminished at 2-months infant age, with much lower proportions of women with a planned or unplanned c-sections breastfeeding exclusively at this period. This discrepancy could be attributed to medical issues experienced postpartum, limited support from family members and healthcare providers as well as work related obligations. ${ }^{16-18}$ This gap between intention to only breastfeed their infant during pregnancy and breastfeeding initiation and continuation in the postnatal period needs to be addressed carefully in future studies. In particular, identifying obstacles based on delivery mode and other factors such as medical problems, women's knowledge around breastfeeding benefits, latching, childcare, job obligations, and lifestyle changes might help future breastfeeding intervention efforts.

Furthermore, the findings of our study indicate that a planned c-section is associated with a significantly lower breastfeeding initiation rate relative to an unplanned c-section or other modes of delivery. Recent studies found similar findings indicating that women with emergency c-sections tend to have a higher likelihood of discontinuing breastfeeding before 12 weeks postpartum compared to those who delivered vaginally. ${ }^{19}$ Other studies that did not distinguish between planned and unplanned c-sections found similar findings in initiating breastfeeding. ${ }^{12,20}$ It has been suggested that lower breastfeeding rates and challenges in women with c-sections might be associated with physiologic mechanisms that affect lactogenesis..$^{21,22}$ For example, research evidence shows that delayed onset of lactation is significantly higher among women with c-section compared to those with vaginally deliveries. ${ }^{22}$ Further, early contact between mothers and infants in the first few hours after birth is important for forming a mother-infant bond that can lead to breastfeeding successful outcomes. However, operative care routines for post caesarean delivery can delay mothers from holding their infants and disrupt bonding between mother and infant; all this negatively affecting breastfeeding initiation. ${ }^{19}$

We also found that women with a vaginally induced delivery had the lowest breastfeeding intensity rate. This is in agreement with previous research findings. ${ }^{1}$ Previous research studies have found that medical procedures during labor, such as labor induction, may often lead women to experience long lasting pain which can delay motherinfant interactions influencing breastfeeding behaviors. ${ }^{1,20}$ Along with mode of delivery, other factors, such as family and clinician opinions, breastfeeding intention as well as work related obligations may influence the mother's infant feeding choice. ${ }^{10}$

Indeed, we found that clinicians and family members were more likely to be supportive of formula only or a combination of breastfeeding and formula among mothers with a planned c-section. This finding coupled with the high levels of disagreement with breastfeeding benefits among this group of women (planned c-section) in the present study may explain their low breastfeeding initiation and duration. Further, we found that women with a planned c-section constituted one of the least likely groups to receive breastfeeding information from a nurse/nurse midwife/nurse practitioner, adding more potential reasons in explaining their poor breastfeeding outcomes. Research evidence shows that healthcare practices during the prenatal and intra partum periods are of extreme importance in successful breastfeeding outcomes. ${ }^{23}$ The findings of the present study clearly show that healthcare providers need to be more supportive of breastfeeding practices among mothers with c-sections, especially those with planned ones. It is known that mothers with a c-section tend to experience longer recovery periods and more medical problems. ${ }^{16,24,25}$ Because of this, it is essential that healthcare providers start conversations around breastfeeding during the prenatal period, when a c-section may be planned. Discussions around infant feeding practices should be addressed not only with the mothers, but also with the immediate family members who might serve as a mechanism of support. A woman is far more likely to initiate and continue breastfeeding in the first few months within a supportive environment. $^{23}$

Our findings show that women with vaginally non-induced deliveries were the least likely to receive breastfeeding information from lactation consultants. Recent research evidence shows that providing breastfeeding information both prenatally and postnatal may be effective in increasing breastfeeding duration and breastfeeding knowledge. ${ }^{26-28}$ Further, we found that women who had a planned c-section were less likely to receive breastfeeding information from family during month 2 postpartum. Research evidence suggest that family and health providers' opinion can have a significant influence on the mother's decision to breastfeed. ${ }^{10}$

Furthermore, women who had an unplanned c- section were more likely to cite medical reasons and to find work or school as major obstacles in initiating breastfeeding during neonatal period. Having more breastfeeding support at workplace by dedicating facilities where mothers can express and store milk has been found to be helpful in increasing breastfeeding initiation and exclusive breastfeeding rates. ${ }^{29}$ Overall, we found that reasoning for not initiating breastfeeding might differ based on mode of delivery. This is an important finding in designing breastfeeding interventions, showing that mode of delivery needs to be considered, and a careful examination of potential reasons for not initiating breastfeeding would be extremely useful in establishing strategies for helping women to overcome these obstacles and have successful breastfeeding outcomes. Also, it is well-known that for first-time mothers the experience of breastfeeding entails major lifestyle changes leading to the need for continuous support with their efforts in initiating and continuing breastfeeding during the first few months. ${ }^{18,30}$

The present study carries several limitations. One of them includes potential response bias due to a decreasing number of women participating in subsequent surveys which can influence breastfeeding information. ${ }^{13}$ We did not account for several important variables such as postpartum health related complications and pain in relation to breastfeeding behaviors due to the lack of such information in the data set. Since the IFPS-II data is not derived from a nationally representative sample, the generalization of our findings is restricted to mothers who have achieved high educational levels and are primarily white. Further, mode of delivery does not distinguish primary vs. repeated cesareans. The validity of some study findings may be limited due to the use of self-reported measures.

\section{Conclusion}

Findings from this study support national and international guidelines regarding infant feeding issues related to breastfeeding initiation, intensity and exclusivity of breastfeeding. It provides evidence that infant feeding practices differ among women who undergo different modes of delivery. Breastfeeding practices among women with a c-section fell short of clinical practice guidelines 
for infant feeding behaviors. Our results suggest that families and clinicians should be educated and encouraged to emphasize the benefits of breastfeeding to mothers while providing support at the same time. Since hospital stay is known to be a critical period for the establishment of breastfeeding, breastfeeding interventions should also focus on improving breastfeeding support in hospitals, particularly among women who have had a planned c-section. Due to our sample being limited to mostly white women with high educational and income levels, future replication studies should include populationbased representation of black and Hispanic mothers.

\section{Acknowledgements}

None.

\section{Conflict of interest}

The authors have no relevant financial disclosures or conflicts of interest in relation to this work.

\section{References}

1. Ahluwalia IB, Li R, Morrow B. Breastfeeding practicesdoes method of delivery matter. Matern Child Health J. 2012;16(2):231-237.

2. Cramton R, Zain-Ul-Abideen M, Whalen B. Optimizing successful breastfeeding in the newborn. Curr Opin Pediatr. 2009;21(3):386-396.

3. Dewey KG, Nommsen-Rivers LA, Heinig MJ, et al. Risk factors for suboptimal infant breastfeeding behavior, Delayed onset of lactation, and excess neonatal weight loss. Pediatrics. 2003;112(3):607-619.

4. Zanardo V, Svegliado G, Cavallin F, et al. Elective cesarean delivery does it have a negative effect on breastfeeding. Birth 2010;37(4):275-279.

5. Kearney MH, Cronenwett LR, Reinhardt R. Cesarean delivery and breastfeeding outcomes. Birth 1990;17(2):97-103.

6. Leung G, Lam TH, Ho LM. Breastfeeding and its relation to smoking and mode of delivery. Obstet Gynecol. 2002;99(5):785-794.

7. Dennis CL. Breastfeeding initiation and duration a 1990-2000 literature review. J Obstet Gynecol Neonatal Nurs. 2003;31(1):12-32.

8. Chezem J, Friesen C, Boettcher J. Breastfeeding knowledge breastfeeding confidence and infant feeding plans effects on actual feeding practices. J Obstet Gynecol Neonatal Nurs. 2003;32(1):40-47.

9. Cakmak H, Kuguoglu S. Comparison of the breastfeeding patterns of mothers who delivered their babies per vagina and via cesarean section: An observational study using the LATCH breastfeeding charting system. Int J Nurs Stud. 2007;44(7):1128-1137.

10. Kornides M, Kitsantas P. Evaluation of breastfeeding promotion support and knowledge of benefits on breastfeeding outcomes. $J$ Child Health Care. 2013;17(3):264-273.

11. Whalen B, Cramton R. Overcoming barriers to breastfeeding continuation and exclusivity. Curr Opin Pediatr. 2010;22(5):655-663.

12. Prior E, Santhakumaran S, Gale C, et al. Breastfeeding after cesarean delivery: a systematic review and meta-analysis of world literature. $\mathrm{Am}$ J Clin Nutr. 2012;95(5):1113-1135.

13. Fein SB, Labiner Wolfe J, Shealy KR, et al. Infant feeding practices study II study methods. Pediatrics. 2008;122(2):S28-S35.
14. Gaffney KF, Kitsantas P, Brito A. Postpartum depression infant feeding practices and infant weight gain at six months of Age. $J$ Pediatr Health Care. 2012;28(1):43-50.

15. Li R, Fein SB, Grummer Strawn LM. Association of breastfeeding intensity and bottle emptying behaviors at early infancy with infants risk for excess weight at late infancy. Pediatrics. 2008;122(2):S77-S84.

16. Borders N, After the Afterbirth a critical review of postpartum health relative to method of delivery. J Midwifery Womens Health. 2006;51(4):242-248.

17. Johnston ML, Esposito N. Barriers and facilitators for breastfeeding among working women in the united states. J Obstet Gynecol Neonatal Nurs. 2007;36(1):9-20.

18. Thulier D Mercer J. Variables associated with breastfeeding duration. $J$ Obstet Gynecol Neonatal Nurs. 2009;38(3):259-268.

19. Hobbs AJ, Mannion CA, McDonald SW, et al. The impact of caesarean section on breastfeeding initiation duration and difficulties in the first four months postpartum. BMC Pregnancy Childbirth. 2016;16(1):90.

20. Rowe Murray HJ, Fisher JRW. Baby friendly hospital practices cesarean section is a persistent barrier to early initiation of breastfeeding. Birth. 2002;29(2):124-131.

21. Evan K, Evans R, Royal R, et al. Effect of caesarean section on breast milk transfer to the normal term newborn over the first week of life. Arch Dis Child Fetal Neonatal Ed. 2003;88(5):F380-F382.

22. Scott JA, Binns CW, Oddy WH. Predictors of delayed onset of lactation. Matern Child Nutr. 2007;3(3):186-193.

23. DiFrisco E, Goodman K, Budin W, et al. Factors associated with exclusive breastfeeding 2 to 4 weeks following discharge from a large, urban, academic medical center striving for baby-friendly designation. $J$ Perinat Educ. 2011;20(1):28-35.

24. Declercq E, Barger M, Cabral HJ, et al. Maternal outcomes associated with planned primary cesarean births compared with planned vaginal births. Obstet gynecol. 2007;109(3):669-677.

25. Miesnik SR, Reale BJ. A review of issues surrounding medically elective cesarean delivery. $J$ Obstet Gynecol Neonatal Nurs. 2007;36(6):605-615.

26. Aksu H, Küçük M, Düzgün G. The effect of postnatal breastfeeding education/support offered at home 3 days after delivery on breastfeeding duration and knowledge a randomized trial. J Matern Neonatal Med. 2011;24(2):354-361.

27. Lumbiganon $\mathrm{P}$, Martis R, Laopaiboon M, et al. Antenatal breastfeeding education for increasing breastfeeding duration. Cochrane Database Syst Rev. 2016;9(11):CD006425.

28. Rosen IM, Krueger MV, Carney LM, et al. Prenatal breastfeeding education and breastfeeding outcomes. MCN, Am J Matern Nurs. 2008;33(5):315-319.

29. Smith JP, McIntyre E, Craig L, et al. Workplace support, breastfeeding and health. Fam matters. 2013;93:58-73.

30. Neifert M, Bunik M. Overcoming clinical barriers to exclusive breastfeeding. Pediatr Clin North Am. 2013;60(1):115-145. 